\title{
The optical control of electronic energy transfer through single and dual auxiliary beams
}

\author{
David S. Bradshaw and David L. Andrews* \\ Nanostructures and Photomolecular Systems, School of Chemical Sciences, \\ University of East Anglia, Norwich NR4 7TJ, United Kingdom
}

\begin{abstract}
The electronic transfer of energy from a donor particle to an acceptor is a mechanism that plays a key role in a wide range of optical and photophysical phenomena. The ability to exert control on this transfer is of immense importance. It now emerges that there are all-optical routes which can be introduced to achieve this very purpose. We demonstrate the possibility of promoting energy transfer, in the optical near field, that is rigorously forbidden (on geometric or symmetric grounds) in the absence of laser light. The effect operates through coupled stimulated Raman scattering by the donoracceptor pair. The absorption of a photon takes place at one particle and stimulated emission at either, coupled with energy transfer between the pair. At this fundamental level, transfer phenomena arise for both single and dual input auxiliary beams. In the latter case the emitted photon may differ from the absorbed photon. In either situation energy transfer will not occur in the absence of auxiliary radiation, if either the donor or acceptor transition is single-quantum forbidden. By engaging input laser light, energy transfer may proceed through two or three quantum allowed transitions. The results for this novel type of optical control suggest transfer efficiency levels comparable to Förster transfer. Many applications are envisaged, chief of which is the potential for all-optical switching.
\end{abstract}

Keywords: Nanostructures, photonics, energy transfer, stimulated Raman, optical switching, photophysics

\section{INTRODUCTION}

As conventional electronic devices approach the practicable size limit, much of the interest in optical switching now focuses on the design of nanoscale assemblies. These operate on a smaller scale but with similar or improved functionality. Many systems have been proposed, including those based on semiconductor quantum wells, ${ }^{1,2}$ electromagnetic induced transparency, ${ }^{3,4}$ optical Kerr effect ${ }^{5,6}$ and surface plasmon polariton media. ${ }^{7,8}$ This paper reports an analysis of a novel all-optical switching mechanism - initially outlined by one of the present authors ${ }^{9,10}$ - based on the optical control of resonance energy transfer (RET) between particles. When RET occurs spontaneously, following the absorption of light, it usually represents the principal process for the inter-particle translation of electronic energy, from the sites of initial optical excitation. However, under suitable conditions such processes of energy transfer can be activated or deactivated by (non-resonant) optical stimulation. The main advantage of this type of photonic switching, compared to the majority of the previously proposed schemes, is the localisation to molecular pairs rather than an ensemble.

In its simplest form, known as Förster transfer, RET involves a relocation of energy from an electronically excited donor $A$ to an acceptor $B$ in its ground state - a mechanism that plays a key role in a wide range of optical and photophysical phenomena. The scheme proposed here, based on optical control of electronic energy transfer, is built around the concept of inhibiting Förster transfer by selection rule constraints, frequency conditions, or geometric architecture. The latter is achieved by apply a condition in which the transition dipole moments of the donor and acceptor, and their mutual displacement vector, are mutually perpendicular. ${ }^{11}$ In this paper we will mainly discuss the exploitation of selection rules, where single-quantum transitions in either $A$ or $B$ are forbidden (thus excluding Förster transfer). By application of an off-resonant laser beam the transfer of energy is activated, through two- or three-quantum allowed transitions, allowing all-optical switching action. In detail, this mechanism entails a coupling of RET with the pairwise absorption and stimulated re-emission of the incident laser, i.e. a coupled stimulated-Raman scattering scheme

*david.andrews@physics.org 


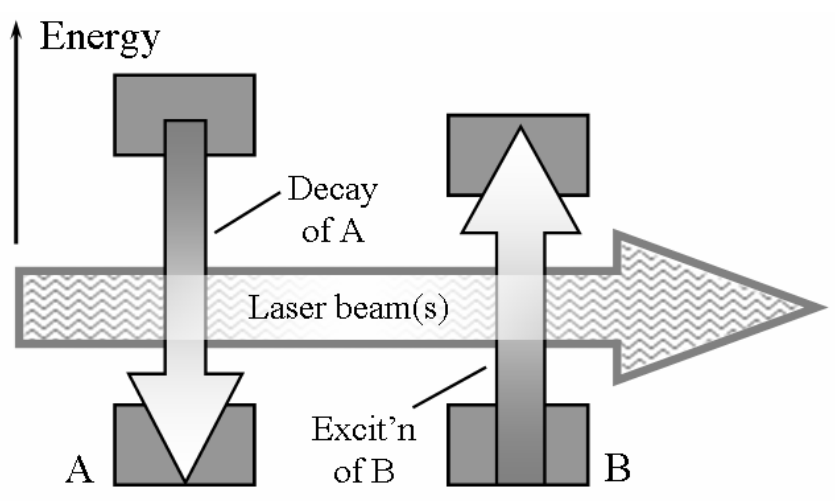

Fig. 1. General schematic of the optical control energy transfer mechanism.

as illustrated by Fig. 1. Furthermore, by application of dual input auxiliary beams - providing conditions where the emitted photon may differ from the absorbed photon - additional effects are anticipated.

In the following we develop the fundamental electrodynamic theory for the optical control of electronic energy transfer, for both single and dual input auxiliary beams (Section 2). The analysis is extended to a two-dimensional square-lattice array arrangement that could be useful for realistic all-optical switches, taking into account the requirement for a multiplicity of such donor-acceptor partners to operate independently, without significant cross-talk (Section 3). Further implementation issues are addressed in the concluding Section 4.

\section{ELECTRODYNAMIC THEORY OF OPTICAL CONTROL}

The optical control of electronic energy transfer for a single input auxiliary beam is first analysed. To specifically determine the efficiency of transfer for this optical control mechanism, a time-dependent perturbation theory method is required. Details of the calculation, which is used to establish the quantum amplitudes of the system, are fully described in refs 12 and 13 and they are not repeated here. As is required for optical switching action based on selection rules, the electronic transitions within donor $A$ or acceptor $B$ are one-photon forbidden. Therefore, no Förster transfer is present and as a result the energy transfer occurs only through a mechanism that engages the auxiliary input beam. In the near-field, the corresponding quantum amplitude involving two-photon transitions is given by;

$$
M_{f i}^{(4)}=\left(\frac{n \hbar c k}{8 \pi \varepsilon_{0}^{2} V R^{3}}\right) e_{i} \bar{e}_{l}\left(\delta_{j k}-3 \hat{R}_{j} \hat{R}_{k}\right)\left(S_{i j}^{A}(k) S_{l k}^{B}(-k)+S_{i j}^{B}(k) S_{l k}^{A}(-k)\right),
$$

where $n$ is the number of photons (proportional to intensity) in the quantization volume $V$, and the implied summation convention for repeated Cartesian tensor indices is employed. Furthermore, $\mathbf{e}$ and $\hbar c k$ denote the polarization vector (an overbar denoting complex conjugation) and energy of the input photon, respectively. Each of the two terms of equation (1), illustrated by the Feynman diagrams of Fig. 2, represents a fourth order photonic interaction event, necessitating deployment of a fourth-order perturbation treatment as indicated by the superscript of $M_{f i}$. Also in (1) is the generalized polarizability, $S_{i j}^{\xi}( \pm k)$, explicitly given by;

$$
S_{i j}^{\xi}( \pm k)=\sum_{r}\left\{\frac{\mu_{i}^{f r} \mu_{j}^{r i}}{\tilde{E}_{r f} \pm \hbar c k}+\frac{\mu_{j}^{f r} \mu_{i}^{r i}}{\tilde{E}_{r i} \mp \hbar c k}\right\} .
$$

Here the transition dipole moments are designated by the shorthand notation $\boldsymbol{\mu}^{x y}=\langle x|\mu| y\rangle$, energy differences are 


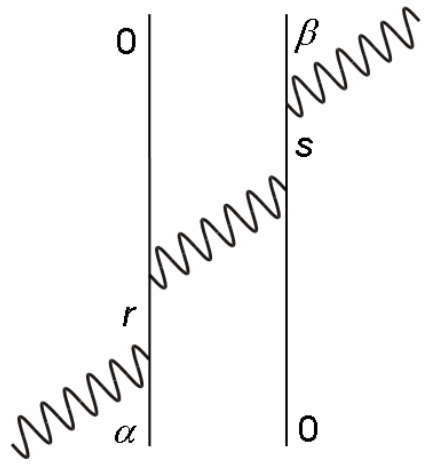

(a)

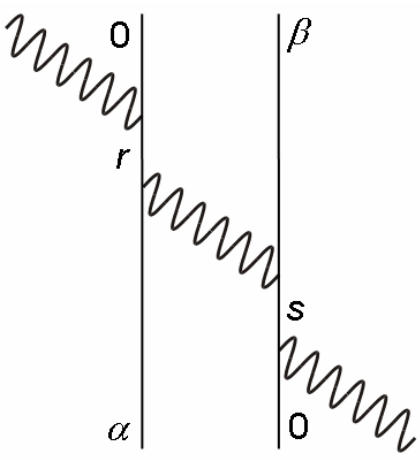

(b)

Fig. 2. Two Feynman diagrams for the optical control mechanism. Here, $|0\rangle$ represents a particle in the ground state; $|\alpha\rangle$ and $|\beta\rangle$ relate to the excited state of the donor (on the left) and acceptor (right), respectively, with $|r\rangle$ and $|s\rangle$ as the corresponding intermediate states. In detail, diagram $(a)$ depicts an instantaneous mechanism involving photon absorption and emission at the donor and acceptor, respectively, with a coupling photon created at the donor and annihilated at the acceptor; thus excitation is transferred from $A$ to $B$. Diagram $(b)$ is a permutation that will achieve an identical final result. Each particle involves two particle-photon interactions and, thus, electronic transitions must be two-photon allowed within both $A$ and $B$.

expressed in the form $E_{x y}=E_{x}-E_{y}$, and tildes denote the inclusion of damping terms necessary if the theory were to be applied under near-resonance conditions ${ }^{14-20}$ - though the present analysis focuses solely on off-resonant photon absorption, and as such the tildes will henceforth be omitted. Further, $f$ signifies the final electronic state of particle $\xi, i$ is the initial state, and $r, s$ are intermediates. To a good approximation, let it be assumed that the sums of equation (2) are limited to the three states that determine the most prominent optical features. These are denoted $|0\rangle,|\sigma\rangle,|\alpha\rangle$ for donor $A$, and $|0\rangle,|\tau\rangle,|\beta\rangle$ for acceptor $B$ - where $|\alpha\rangle$ and $|\beta\rangle$ are the levels between which energy transfer occurs. It is also expedient to select a frequency for the input radiation which has a resonance offset with respect to the positioning of these levels, a condition expressible as $E_{\sigma \alpha}=\hbar c k+\Delta E_{A}$, where $\Delta E_{A}$ is a non-zero energy with magnitude significantly lower than a typical transition energy. An expression of similar form, $E_{\tau \beta}=\hbar c k+\Delta E_{B}$, is assumed for $B$. The outcome of applying these conditions is that one summand is significantly larger in magnitude than the rest. Hence, equation (1) becomes:

$$
M_{f i}^{(4)}=\left(\frac{n \hbar c k\left(\mathbf{e} \cdot \boldsymbol{\mu}^{\sigma \alpha}\right)\left(\overline{\mathbf{e}} \cdot \boldsymbol{\mu}^{\beta \tau}\right)}{8 \pi \varepsilon_{0}^{2} V R^{3} \Delta E_{A} \Delta E_{B}}\right)\left(\boldsymbol{\mu}^{0 \sigma} \cdot \boldsymbol{\mu}^{\tau 0}-3\left(\boldsymbol{\mu}^{0 \sigma} \cdot \hat{\mathbf{R}}\right)\left(\boldsymbol{\mu}^{\tau 0} \cdot \hat{\mathbf{R}}\right)\right)
$$

Next, to proceed to the geometric conditions, we introduce a Cartesian basis in which the donor-acceptor displacement vector $\mathbf{R}$ is identified with the $z$-direction. The vectors $\hat{\boldsymbol{\mu}}_{A} \equiv \hat{\boldsymbol{\mu}}^{0 \alpha}$ and $\hat{\boldsymbol{\mu}}_{B} \equiv \hat{\boldsymbol{\mu}}^{\beta 0}$ are chosen unambiguously as being directed in the $\hat{\mathbf{i}}$ and $\hat{\mathbf{j}}$ directions respectively and, by a judicious choice which is fully justifiable on symmetry grounds, $\hat{\boldsymbol{\mu}}^{0 \sigma}=\hat{\mathbf{k}}$ and $\hat{\boldsymbol{\mu}}^{\sigma \alpha}=\hat{\mathbf{j}}$. Also, $\hat{\boldsymbol{\mu}}^{\tau 0}=\hat{\mathbf{k}}$ and $\hat{\boldsymbol{\mu}}^{\beta \tau}=-\hat{\mathbf{i}}$ are taken. For convenience $A$ and $B$ are chosen to belong to identical symmetry groups, although the same calculational method allows for systems where this is not necessarily the case. Therefore, employing this configuration, equation (3) becomes;

$$
M_{f i}^{(4)}=\left(\frac{-n \hbar c k|\mu|^{4} \kappa^{\prime}}{8 \pi \varepsilon_{0}^{2} V r^{3} \Delta E_{A} \Delta E_{B}}\right) \sin ^{2} \phi \cos \theta \sin \theta,
$$


where the orientation factor is $\kappa^{\prime}=\left(R^{2}-3 r^{2}\right) / R^{2}=-2$ given that $\mathbf{R}=r \hat{\mathbf{k}}, r$ being the displacement of $B$ from $A$. Furthermore, the given angles denote the orientation of $\mathbf{e}$ in spherical coordinates (with the input photon polarization taken as linear). In addition, all transition dipole moments will have similar magnitude - thus, for simplicity, assumed to be equal. Equation (4) clearly delivers a non-zero result, unlike the now precluded Förster transfer. The time-dependent probability, $P(t)$, that the energy transfer process can proceed is secured from the Fermi's Golden Rule, here expressible in the form;

$$
P(t)=\frac{2 \pi \rho_{f}}{\hbar} \int_{0}^{t}\left|M_{f i}^{(2)}+M_{f i}^{(4)}+\ldots\right|^{2} \mathrm{~d} t
$$

with higher-order quantum amplitudes rapidly diminishing in magnitude. Here, the second-order contribution $M_{f i}^{(2)}$ corresponds to RET - a null quantity for cases described in this paper - and $\rho_{f}$ is the density of states. Inserting (4) into (5) gives the expression;

$$
P(t)=\frac{4 K C^{2} J^{\prime}}{r^{6}} \sin ^{4} \phi \cos ^{2} \theta \sin ^{2} \theta
$$

where the variables are defined as $K=|\mu|^{4} \rho_{f} / 8 \pi \varepsilon_{0}^{2} \hbar, \quad C=|\mu|^{2} / 2 c \varepsilon_{0} \Delta E_{A} \Delta E_{B}$ and $J^{\prime}=\int_{0}^{t} I^{2}(t) \mathrm{d} t$, in which $I(t) \equiv n \hbar c^{2} k / V$ is the irradiance. It is noteworthy that with $\phi=0^{\circ}, \theta=0^{\circ}$ or $\theta=90^{\circ}$, no energy transfer to the acceptor occurs. Although not shown here, as a result of the condition that $A$ and $B$ have identical symmetry, there is a further mechanism that involves three-photon transitions at one particle (corresponding to three particle-photon events) and one-photon transition at the other (see Fig. 3). The intricate analysis for this three-photon allowed mechanism is detailed within refs 21 and 22.

By extension of these principles, a dual-beam configuration can now be considered. Specifically, the earlier expressions may be recast to allow for the case where the $A-B$ system is simultaneously subject to the confluence of two beams of off-resonant laser radiation. By following the same procedure as above, $P(t)$ will be equivalent to equation (6) except for $J^{\prime}=\int_{0}^{t} I_{1}(t) I_{2}(t) \mathrm{d} t$, i.e. both intensities of the incident and emission beams are included. Further, the values $\Delta E_{A}$ and $\Delta E_{B}$ are more easily manipulated. Thus, an advantage of dual auxiliary beams over a single-beam set-up is the greater number of independently controllable experimental parameters, resulting in enhanced control of transfer

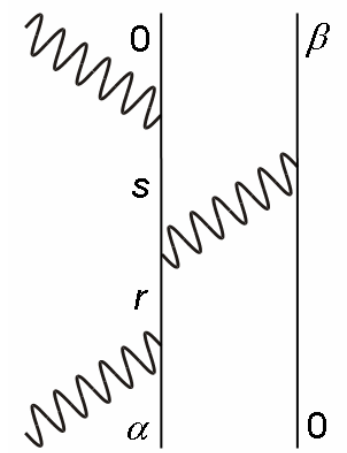

(a)

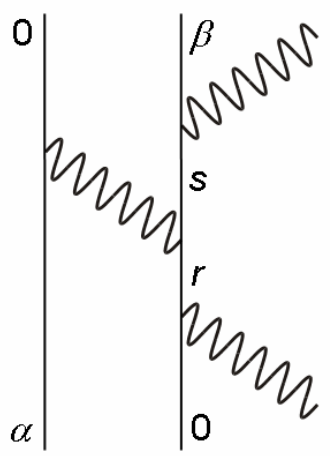

(b)

Fig. 3. Two further Feynman diagrams for the optical control mechanism. Here, diagram $(a)$ depicts an instantaneous mechanism involving photon absorption and emission at a donor, with a coupling photon created at the donor and annihilated at the acceptor; thus excitation is transferred from $A$ to $B$. Diagram $(b)$ is a permutation, where photon absorption and emission occurs at the acceptor. At one particle three particle-photon interactions occur (the other particle one interaction) and, thus, electronic transitions must be three-photon allowed at this particle (one-photon allowed at the other). 


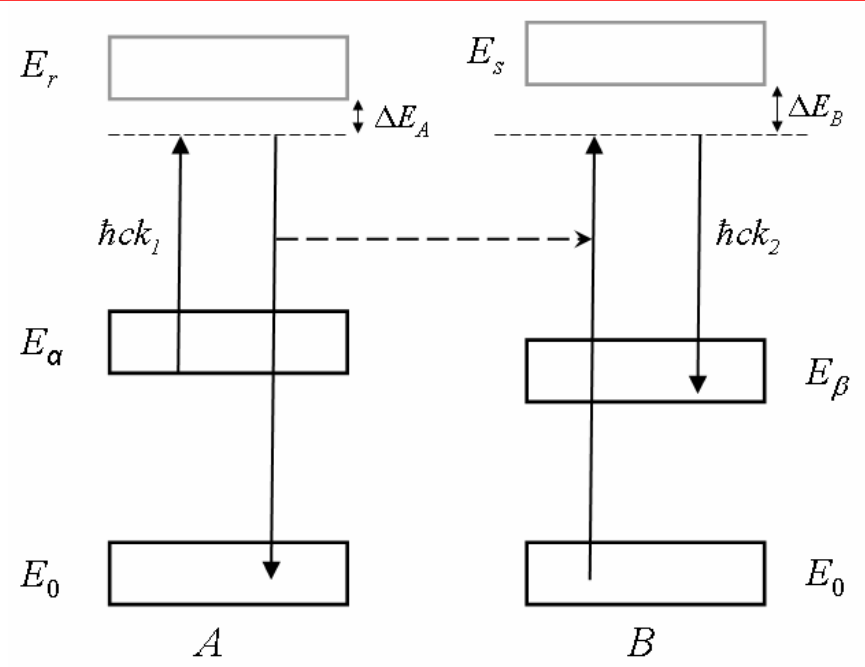

Fig. 4. Energetics scheme for optically controlled transfer of energy from $A$ to $B$. Solid-head arrows denote four transitions coupling the donor decay $|\alpha\rangle \rightarrow|0\rangle$ and acceptor excitation $|0\rangle \rightarrow|\beta\rangle$, laser interactions denoted by the photon energies. Dotted lines denote virtual states, the closest real states $\mathrm{E}_{r}$ and $\mathrm{E}_{s}$ offset in energy by $\Delta E_{A}$ and $\Delta E_{B}$. The dashed arrow signifies energy transfer.

efficiency between the $A-B$ pair. A further benefit is the presence of optical switching, despite a significant mismatch between $|\alpha\rangle$ and $|\beta\rangle$. This is due to the compensation of this mismatch by the difference in frequency of the two beams, resulting in the supply of a quantum of energy that enables excitation transfer to occur between the particles (Fig. 4). In detail, the incident photon of energy $\hbar c k_{1}$ is absorbed at $A$ and a further photon with $\hbar c k_{2}$ is emitted from $B$, the difference $\left(\hbar c k_{1}-\hbar c k_{2}\right)$ equates to the mismatch between $|\alpha\rangle$ and $|\beta\rangle .^{23}$ A related advantage is that spontaneous RET is energetically forbidden (especially with $E_{\alpha}<E_{\beta}$ ) and, therefore, the selection rules and geometric condition (briefly discussed earlier) need not be applied - i.e. practical implementation is perhaps made much easier.

\section{TWO-DIMENSIONAL SQUARE-LATTICE ARRAYS}

To extend and develop the single-beam donor-acceptor model of the last section into that of a potential all-optical switch, we now investigate the case of a parallel pair of two-dimensional square-lattice arrays - each of which is composed of equally spaced, identical particles (Fig. 5). These are constructed such that each constituent particle of the donor array directly corresponds to a counterpart within the acceptor array; these pairs are given the coordinates $u$ and $v$ (integer values) and are displaced, one from each other, by $r \hat{\mathbf{k}}$. Furthermore, all particles in the donor and acceptor arrays are orientated in the $\hat{\mathbf{i}}$ and $\hat{\mathbf{j}}$ directions, respectively, so that they are consistent with the configurational condition introduced in the previous section. Thus, particles in each array are labelled $(u, v)$ and the corresponding displacement from the origin is $\mathbf{R}=u \hat{\mathbf{i}}+v \hat{\mathbf{j}}+r \hat{\mathbf{k}}$, with $l$ as the lattice constant. It is expedient to focus on processes by means of which energy can transfer from any one specific excited donor (for convenience located at $u=0$ ) to any other particle. First, consider energy relocation to an arbitrary particle within the acceptor array. To find an expression for $P(t)$, noting Förster transfer is again excluded, the following equation is determined;

$$
M_{f i}^{(4)}=\left(\frac{-n \hbar c k|\mu|^{4}}{8 \pi \varepsilon_{0}^{2} l^{3} V \Delta E_{A} \Delta E_{B}}\right)\left(\frac{u^{2}+v^{2}-2 r^{\prime 2}}{\left(u^{2}+v^{2}+r^{\prime 2}\right)^{\frac{5}{2}}}\right) \sin ^{2} \phi \cos \theta \sin \theta,
$$

as $\kappa^{\prime}=\left(u^{2}+v^{2}-2 r^{\prime 2}\right) /\left(u^{2}+v^{2}+r^{\prime 2}\right)$, in which $r^{\prime}=r / l$ is the aspect ratio. It is readily verified that equation (7) 


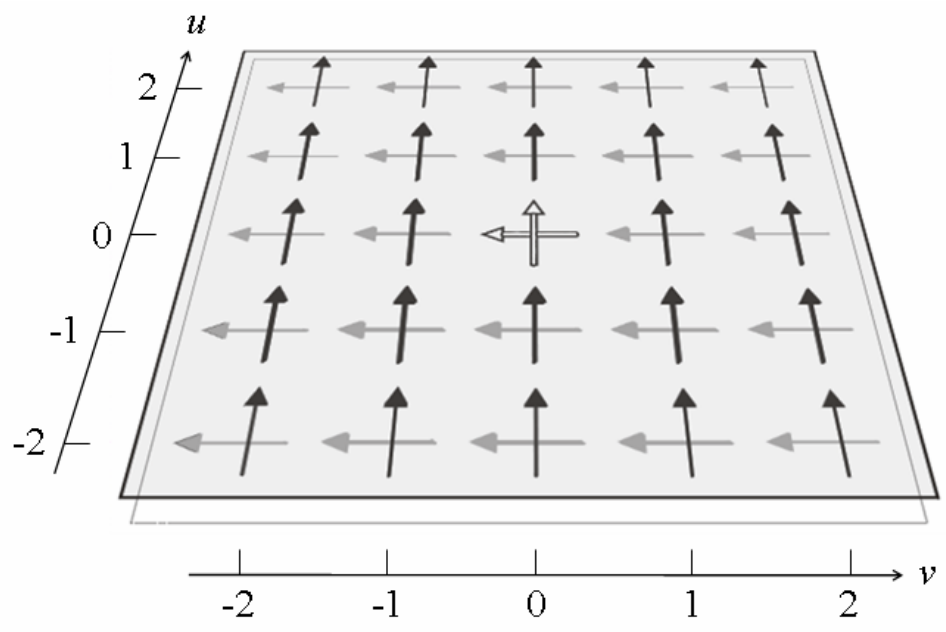

Fig. 5. Structure of the two-dimensional square-lattice arrays, viewed from above. Both lie in the $i j$-plane, with all donor transition moments (black) in the upper array parallel to the $i$-axis, and all acceptor transition moments (gray) in the lower array parallel to the $j$-axis. The open arrows represent one excited donor and its counterpart acceptor.

reduces to (4), i.e. the result for a directly opposing donor-acceptor pair, on setting $u=0$ and $v=0$. Employing equation (7), with (5), leads to the following result;

$$
P(t)=\frac{K C^{2} J^{\prime}\left(u^{2}+v^{2}-2 r^{\prime 2}\right)^{2}}{l^{6}\left(u^{2}+v^{2}+r^{\prime 2}\right)^{5}} \sin ^{4} \phi \cos ^{2} \theta \sin ^{2} \theta .
$$

The second case to consider is a transfer of energy from one initially excited donor particle to another within the donor array. The resulting expression is analogous to equation (8), but with $r^{\prime}$ taken as zero.

To quantify the extent to which a device based on the parallel array geometry could faithfully transmit information (constituted by the pattern of excitations in the donor array) to the acceptor array, we can consider a twinarray transfer fidelity: this is defined as the efficiency of direct energy relocation, from an excited donor to its designated partner in the acceptor array, to the summed efficiencies for transfer to any other particles within either array. From calculations based on the modest parameter values $I=1 \times 10^{10} \mathrm{~W} \mathrm{~cm}^{-2}, \Delta E=3 \times 10^{-20} \mathrm{~J},|\mu|=1 \times 10^{-29} \mathrm{C} \mathrm{m}$, and with $\phi=90^{\circ}$ and $\theta=45^{\circ}$, the dependence of the result on the aspect ratio is determined and graphically depicted as plots of $P(t)$ for different transfer destinations. In this representation, energy transfer outside the range $(-10 \leq u \leq 10)$ and $(-10 \leq$ $v \leq 10)$ is negligible. As the plots within Fig. 6 illustrate, the transfer of energy from the excited donor to the corresponding particle in the acceptor array greatly dominates all other transfer routes for any aspect ratio, $r^{\prime}$, up to $\sim 0.6$. Specifically, no less than $95 \%$ of the total excitation is transported to the required destination. As might be anticipated, this transfer fidelity is increasingly compromised when the aspect ratio is increased - see fig. 6 (inset). It is interesting to note that the fidelity is independent of the laser intensity. The dual auxiliary beam configuration will give the same results and, thus, will not affect the destination of the excitation to any greater extent than the single-beam set-up. Therefore, no additional benefits are determined for an array system, outside those given previously, with the employment of two input beams. 


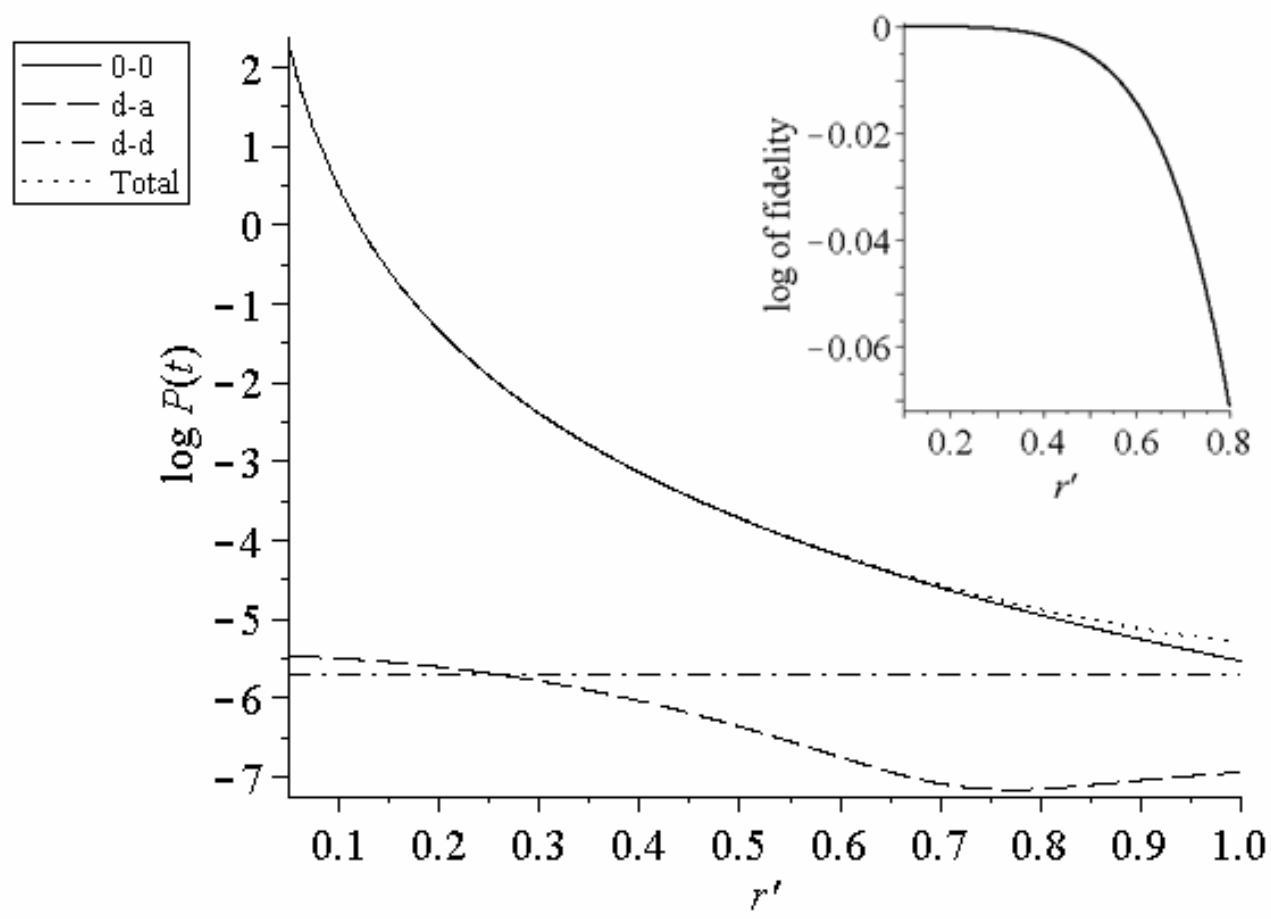

Fig. 6. Plot of $\log P(t)$, where $P(t)$ is the time dependent probability, against the aspect ratio, $r^{\prime}$, for optical transfer from an excited particle in the donor square-lattice array to the required destination in the acceptor square array (0-0); also depicted are the 'cross talk' probabilities for transfer to another particle in either the acceptor (d-a) or the donor (d-d) array, and the sum of all three transfer possibilities (Total). Inset: difference between logarithms of the 0-0 and the sum probabilities for various $r^{\prime}$, signifying on a logarithmic scale the transfer fidelity; on the ordinate axis each -0.01 increment corresponds to $2.3 \%$ loss. Here, the intensity $I$ of the input laser is $1 \times 10^{10} \mathrm{~W} \mathrm{~cm}^{-2}$.

\section{CONCLUSION}

This paper has investigated an optical control process that has potential for a variety of device implementations. The distinct features of two-dimensional nano-array structures have been analyzed in detail, and their relative efficiencies, transfer fidelities and practicality have been critically assessed. Calculations have shown that this optically controlled mechanism for energy transfer has similar efficiency levels to Förster transfer, even for modest pulsed laser intensities of around $10^{12} \mathrm{~W} \mathrm{~cm}^{-2}$. The optical switching mechanism has been explored for both single and dual input auxiliary beams. The advantages of the dual-beam configurations have been detailed - in short; there are more independently controllable experimental parameters, optical switching still occurs despite a major mismatch between $|\alpha\rangle$ and $|\beta\rangle$ and the selection rules to preclude RET need not be applied. The dual-beam configuration acts as a logical AND gate rather than an optical transistor. Nonetheless, there are a number of disadvantages, including; a workable device will be more intricate to manufacture, and two laser pulses of different frequencies will require synchronisation. Practically, the latter will probably involve the use of a beam-splitter to derive two pulse trains from a given mode-locked pump source, one train will pass through a frequency-shifting elements and recombine with the other train ensuring temporal overlap of pulses. Ongoing research will explore the obvious switching, logic gate and transistor attributes, but the aspect that might hold the greatest promise for optical communications and data transmission is the capacity of such a system to act as an optical buffer. 


\section{ACKNOWLEDGEMENTS}

We wish to thank the Leverhulme Trust for providing the financial support for this research.

\section{REFERENCES}

1. G. W. Cong, R. Akimoto, K. Akita, T. Hasama, and H. Ishikawa, "Low-saturation-energy-driven ultrafast alloptical switching operation in $(\mathrm{CdS} / \mathrm{ZnSe}) / \mathrm{BeTe}$ intersubband transition", Opt. Express 15, pp. 12123-12130, 2007.

2. Y. Fedoryshyn, P. Strasser, P. Ma, F. Robin, and H. Jäckel, "Optical waveguide structure for an all-optical switch based on intersubband transitions in InGaAs/AlAsSb quantum wells", Opt. Lett. 32, pp. 2680-2682, 2007.

3. B. S. Ham and P. R. Hemmer, "Coherence switching in a four-level system: Quantum switching", Phys. Rev. Lett. 84, pp. 4080-4083, 2000.

4. P. Bermel, A. Rodriguez, S. G. Johnson, J. D. Joannopoulos, and M. Soljacic, "Single-photon all-optical switching using waveguide-cavity quantum electrodynamics", Phys. Rev. A 74, 043818, 2006.

5. N. Moll, R. Harbers, R. F. Mahrt, and G. L. Bona, "Integrated all-optical switch in a cross-waveguide geometry", Appl. Phys. Lett. 88, 171104, 2006.

6. M. R. Singh and R. H. Lipson, "Optical switching in nonlinear photonic crystals lightly doped with nanostructures", J. Phys. B 41, 015401, 2008.

7. G. A. Wurtz, R. Pollard, and A. V. Zayats, "Optical bistability in nonlinear surface-plasmon polaritonic crystals", Phys. Rev. Lett. 97, 057402, 2006.

8. D. E. Chang, A. S. Sorensen, E. A. Demler, and M. D. Lukin, "A single-photon transistor using nanoscale surface plasmons", Nature Physics 3, pp. 807-812, 2007.

9. D. L. Andrews and R. G. Crisp, "Optically activated energy transfer: array implementation", J. Opt. A 8, pp. S106-S112, 2006.

10. D. L. Andrews and R. G. Crisp, "Theory of directed electronic energy transfer", J. Fluoresc. 16, pp. 191-199, 2006.

11. D. L. Andrews and D. S. Bradshaw, "Virtual photons, dipole fields and energy transfer: a quantum electrodynamical approach", Eur. J. Phys. 25, pp. 845-858, 2004.

12. $\quad$ P. Allcock, R. D. Jenkins, and D. L. Andrews, "Laser assisted resonance energy transfer", Chem. Phys. Lett. 301, pp. 228-234, 1999.

13. $\quad$ P. Allcock, R. D. Jenkins, and D. L. Andrews, "Laser-assisted resonance-energy transfer", Phys. Rev. A 61, $023812,2000$.

14. D. L. Andrews, S. Naguleswaran, and G. E. Stedman, "Phenomenological damping of nonlinear-optical response tensors", Phys. Rev. A 57, pp. 4925-4929, 1998.

15. D. L. Andrews, L. C. D. Romero, and G. E. Stedman, "Polarizability and the resonance scattering of light: Damping sign issues", Phys. Rev. A 67, 055801, 2003.

16. P. W. Milonni and R. W. Boyd, "Influence of radiative damping on the optical-frequency susceptibility", Phys. Rev. A 69, 023814, 2004.

17. R. Loudon and S. M. Barnett, "Theory of the linear polarizability of a two-level atom", J. Phys. B 39, pp. S555S563, 2006.

18. P. R. Berman, R. W. Boyd, and P. W. Milonni, "Polarizability and the optical theorem for a two-level atom with radiative broadening", Phys. Rev. A 74, 053816, 2006.

19. S. Mukamel, "Causal versus noncausal description of nonlinear wave mixing: Resolving the damping-sign controversy", Phys. Rev. A 76, 021803, 2007.

20. I. Bialynicki-Birula and T. Sowinski, "Quantum electrodynamics of qubits", Phys. Rev. A 76, 062106, 2007.

21. D. L. Andrews, R. G. Crisp, and S. Li, "Single and dual beam optical switching of resonance energy transfer", J. Chem. Phys. 127, $174702,2007$.

22. D. S. Bradshaw and D. L. Andrews, "Optically controlled resonance energy transfer: Mechanism and configuration for all-optical switching", J. Chem. Phys., in press.

23. D. L. Andrews, "Optically switched energy transfer: Twin-beam off-resonance control", Phys. Rev. Lett. 99, 023601, 2007. 